\title{
On the Nature of "Strong" Bogoliubov Transformations for Fermions *
}

\author{
Gilles Labonté $\star \star$ \\ Theoretical Physics Institute, University of Alberta, Edmonton, Canada
}

Received November 6, 1973

\begin{abstract}
Properties of the unitary operator implementing a general Bogoliubov transformation on a system of fermions are examined. The nature of this operator (and that of the transformed vacuum state) is exhibited for the case in which the operators appearing in the "diagonal" part of the transformation do not have an inverse. This case turns out to be very different from either the case where the inverses exist or the case with Bogoliubov transformations on systems of bosons. In particular, such transformations can be unitarily implementable in the initial Fock-Hilbert space even though the new vacuum is orthogonal to the initial vacuum. Particles are then created with certainty (i.e. probability 1) in the initial vacuum state and even the new charge operator could be different from the initial one.
\end{abstract}

\section{Introduction}

In many problems where a quantized field is acted upon by an external field, the differential equation of motion for the quantized field operator valued distribution is linear and homogeneous. This is the case, for example, when a Dirac electron-positron field, $\psi(t, x)$, is modified through minimal coupling with a classical electromagnetic field $A(t, x)$ according to

$$
(-i \gamma \cdot \partial+m) \psi(t, x)=e \gamma \cdot A(t, x) \psi(t, x) .
$$

In such problems, one is interested in knowing about the existence and nature of the $S$-matrix or of the unitary time evolution operator at finite times for the field operator variables or the one for the energy quanta creation and annihilation operator variables. Thus one is led to examine linear homogeneous relations, of the type described in Eq. (1.1) below ${ }^{1}$,

* This work is part of the author's Ph. D. thesis done at the University of Alberta and was supported by the National Research Council of Canada and the Department of Advanced Education of the Government of Alberta.

$\star \star$ Research supported in part by Air Force Office of Scientific Research under Contract F 44620-71-C-0108 (USA).

${ }^{1}$ We will study here explicitly theories with distinct particles and antiparticles. The results for theories with only one kind of particle can be obtained in a similar fashion. They will be mentioned at the end. 
between different creation and annihilation operators i.e. to examine Bogoliubov transformations. (For more details on how exactly these transformations come about in external field problems, see for example: Part V of Friedrichs' book [1], Capri [2], Schroer, Seiler, and Swieca [3], and Seiler [4].) Stated more precisely, one needs the answers to the following questions: when is a Bogoliubov transformation unitarily implementable in the Fock-Hilbert space determined by the action of the untransformed creation operators on the untransformed vacuum? and what is the nature (i.e. its expression in terms of untransformed operators for example) of the unitary operator implementing such a transformation?

In the case of boson fields, the answers to these questions can be found in the Sections 23, 24, 25 of the Part V of Friedrichs' book [1] and in theorems proved in the articles by Shale [5] and Seiler [4]. The answer to the first question is: there exists a unitary operator $T$, such that $T^{\dagger}$ \{all creation operators $\left.b^{\dagger}, d^{\dagger}\right\} T=$ all creation operators $B^{\dagger}, D^{\dagger}$ which are related to the $b^{\dagger}, d^{\dagger}$ according to Eq. (1.1)\}, if and only if the operators $M_{i}^{\prime}$ 's appearing in (1.1) are such that

$$
\left\|M_{1}^{-1} M_{2}\right\|_{\text {H.S. }}^{2}=\int d \alpha d \beta\left|\int d \gamma M_{1}^{-1}(\gamma, \alpha) M_{2}(\gamma, \beta)\right|^{2}<\infty .
$$

(In particular, the condition $\left\|M_{2}\right\|_{\text {H.S. }}<\infty$ is sufficient for the existence of $T$.) As for the unitary operator $T$, different forms for it are given by Friedrichs. It is always such that the transformed vacuum state $\left|\phi_{0}\right\rangle$ is

$$
\left|\phi_{0}\right\rangle=\eta \exp -\int d \alpha d \beta b^{\dagger}(\alpha)\left(M_{1}^{-1} M_{2}\right)(\alpha, \beta) d^{\dagger}(\beta)|0\rangle
$$

where $|0\rangle$ is the initial vacuum and $\eta$ a finite normalization constant. For theories with only one kind of particle, Eq. (1.1) is replaced by

$$
B(\gamma)=\int d \alpha M_{1}(\gamma, \alpha) b(\alpha)+\int d \alpha M_{2}(\gamma, \alpha) b^{\dagger}(\alpha)
$$

and the necessary and sufficient condition for the existence of $T$ is also $\left\|M_{1}^{-1} M_{2}\right\|_{\text {H.S. }}<\infty$. The transformed vacuum is then

$$
\left|\phi_{0}\right\rangle=\eta \exp -\int d \alpha d \alpha^{\prime} b^{\dagger}(\alpha)\left(M_{1}^{-1} M_{2}\right)\left(\alpha, \alpha^{\prime}\right) b^{\dagger}\left(\alpha^{\prime}\right)|0\rangle .
$$

For fermion fields, one remarks that it is usually not clear in the literature on external field theory what conditions are necessary and sufficient for the existence of a unitary operator implementing the transformation. However, it was mentioned by Bongaarts [6] and Seiler [4] that such conditions can be found in theorems by Shale and Stinespring [7]. (We will explain these conditions below.) The lack of a 
precise result in the other treatments arises from the fact that it is possible for the operators $M_{1}$ and $M_{4}^{*}$ appearing in (1.1) not to have an inverse and this possibility has not been analyzed completely.

The fermion transformations, indeed, have the particularity that the operators $M_{1}$ and $M_{4}^{*}$ are not guaranteed, by only the relations (1.7) and (1.9) below, to have an inverse. This was guaranted in the case of bosons because a minus sign appeared in front of $M_{2} M_{2}^{*}$ and $M_{3} M_{3}^{*}$ in the corresponding relations. Those transformations where $M_{1}^{-1}$ and $\left(M_{4}^{*}\right)^{-1}$ exist can be treated in a similar manner as in the boson case: this was done by Friedrichs. He gave different forms for the corresponding unitary operators and these are in fact of the same type as those for bosons. The question of existence of the unitary operator in the general case was completely settled by Shale and Stinespring, as mentioned already. The result is: there exists a unitary operator $T$ implementing a Bogoliubov transformation [as (1.1) below] in the Fock-Hilbert space determined by the untransformed operators and the untransformed vacuum if and only if $\left\|M_{2}\right\|_{\text {H.S. }}<\infty$ and $\left\|M_{3}\right\|_{\text {H.S. }}<\infty$. $\left[M_{2}\right.$ and $M_{3}$ being the operators appearing in the off-diagonal part of (1.1).]

Our own contribution in the present note consists in giving explicit expressions for the transformed vacuum state and for the unitary operator implementing the transformation when $M_{1}$ and $M_{4}^{*}$ do not have an inverse (these are the transformations which we refer to as "strong" transformations).

The method used to find such expressions yields, at the same time, an alternative proof (by construction) of the above-mentioned result concerning the existence conditions. We will proceed as follows.

We first explain the notation which we will use; it is essentially the one used in the external field problem where the Fermi particles and antiparticles are different. The transformation (1.1) will be considered to be a general transformation of the type encountered in such problems.

We then prove that the conditions $\left\|M_{2}\right\|_{\text {H.S. }}<\infty$ and $\left\|M_{3}\right\|_{\text {H.S. }}<\infty$ are necessary for the existence, in the initial Fock-Hilbert space, of a vacuum state annihilated by all the transformed annihilation operators. The existence of such a state is certainly necessary for the two sets of creation and annihilation operators (transformed and untransformed) to be unitarily related. The above conditions on $M_{2}$ and $M_{3}$ are therefore necessary conditions for the unitarity implementability of the Bogoliubov transformation (1.1).

Supposing that these conditions are satisfied, we then decompose the general transformation in a product of simpler transformations. The properties of the unitary operator, in the general case, are thus made evident. It will be constructed easily with the help of the results given by Friedrichs for the case of "weak" transformations. 


\section{Definition}

We consider transformations of the type

$$
\begin{aligned}
B(\gamma) & =\int d \alpha M_{1}(\gamma, \alpha) b(\alpha)+\int d \beta M_{2}(\gamma, \beta) d^{\dagger}(\beta) \\
D^{\dagger}(\lambda) & =\int d \alpha M_{3}(\lambda, \alpha) b(\alpha)+\int d \beta M_{4}(\lambda, \beta) d^{\dagger}(\beta)
\end{aligned}
$$

where the four infinite sets of indices $\{\alpha\},\{\beta\},\{\gamma\}$, and $\{\lambda\}$ are not necessarily the same and each one can be discrete or continuous or have both continuous and discrete elements. Integrals, like

$$
\int d \alpha, \text { mean } \int_{\text {(over continuous } \left.\alpha^{\prime} s\right)} d \alpha+\sum_{\substack{\left.\alpha \\ \text { (over dicrete } \alpha^{\prime} s\right)}}
$$

In Eq. (1.1), $b(\alpha)$ and $d^{\dagger}(\beta)$ are operators such that with their adjoints they satisfy the anticommutation relations

$$
\left\{b(\alpha), b^{\dagger}\left(\alpha^{\prime}\right)\right\}=\delta_{\alpha \alpha^{\prime}} \quad\left\{d(\beta), d^{\dagger}\left(\beta^{\prime}\right)\right\}=\delta_{\beta \beta^{\prime}}
$$

with all other anticommutators vanishing. It is understood that for continuous values of the indices, these are operator-valued distributions i.e. they are well defined operators only when smeared with a square integrable function of the index.

They are defined on the Hilbert space $\mathscr{H}$ consisting of states

$$
|\psi\rangle=\sum_{n=0}^{\infty} \sum_{m=0}^{\infty} \frac{1}{\sqrt{n ! m !}} \int \boldsymbol{d} \boldsymbol{\alpha}_{n} \boldsymbol{d} \boldsymbol{\beta}_{m} \psi^{n m}\left(\boldsymbol{\alpha}_{n} ; \boldsymbol{\beta}_{m}\right)\left|\boldsymbol{\alpha}_{n} ; \boldsymbol{\beta}_{m}\right\rangle
$$

such that

$$
\langle\psi \mid \psi\rangle=\sum_{n=0}^{\infty} \sum_{m=0}^{\infty} \int \boldsymbol{d} \boldsymbol{\alpha}_{n} \boldsymbol{d} \boldsymbol{\beta}_{m}\left|\psi^{n m}\left(\boldsymbol{\alpha}_{n} ; \boldsymbol{\beta}_{m}\right)\right|^{2}<\infty
$$

and where by definition

$$
\begin{aligned}
\left|\alpha_{n} ; \boldsymbol{\beta}_{m}\right\rangle= & \left|\alpha_{1}, \alpha_{2}, \ldots, \alpha_{n} ; \beta_{1}, \beta_{2}, \ldots, \beta_{m}\right\rangle \\
= & b^{\dagger}\left(\alpha_{1}\right) b^{\dagger}\left(\alpha_{2}\right) \ldots b^{\dagger}\left(\alpha_{n}\right) d^{\dagger}\left(\beta_{1}\right) d^{\dagger}\left(\beta_{2}\right) \ldots \\
& \ldots d^{\dagger}\left(\beta_{m}\right)|0\rangle
\end{aligned}
$$

and the vacuum state $|0\rangle$ is such that

$$
b(\alpha)|0\rangle=0 \forall \alpha \quad d(\beta)|0\rangle=0 \forall \beta .
$$

We will use the notation

$$
\left(\frac{\boldsymbol{\alpha}_{n}}{\hat{i}}\right)=\left(\alpha_{1}, \alpha_{2}, \ldots, \alpha_{i-1}, \alpha_{i+1}, \ldots, \alpha_{n}\right) .
$$


The anticommutation relations of the operators $B, D$ will be of the same type as for the operators $b, d$ when the following relations hold between the $M_{i}^{\prime}$ s:

$$
\begin{gathered}
\int d \alpha M_{1}(\gamma, \alpha) M_{1}^{*}\left(\gamma^{\prime}, \alpha\right)+\int d \beta M_{2}(\gamma, \beta) M_{2}^{*}\left(\gamma^{\prime}, \beta\right)=\delta_{\gamma \gamma^{\prime}} \\
\int d \alpha M_{3}(\lambda, \alpha) M_{3}^{*}\left(\lambda^{\prime}, \alpha\right)+\int d \beta M_{4}(\lambda, \beta) M_{4}^{*}\left(\lambda^{\prime}, \beta\right)=\delta_{\lambda \lambda^{\prime}} \\
\int d \alpha M_{1}(\gamma, \alpha) M_{3}^{*}(\lambda, \alpha)+\int d \beta M_{2}(\gamma, \beta) M_{4}^{*}(\lambda, \beta)=0 .
\end{gathered}
$$

By demanding that the transformation (1.1) had an inverse and using the relations (1.7) together with the similar relations for the inverse transformation, we can see that the inverse is the adjoint of the transformation (1.1), i.e.

with

$$
\begin{aligned}
b(\alpha) & =\int d \gamma M_{1}^{*}(\gamma, \alpha) B(\gamma)+\int d \lambda M_{3}^{*}(\lambda, \alpha) D^{\dagger}(\lambda) \\
d^{\dagger}(\beta) & =\int d \gamma M_{2}^{*}(\gamma, \beta) B(\gamma)+\int d \lambda M_{4}^{*}(\lambda, \beta) D^{\dagger}(\lambda)
\end{aligned}
$$

$$
\begin{gathered}
\int d \gamma M_{1}(\gamma, \alpha) M_{1}^{*}\left(\gamma, \alpha^{\prime}\right)+\int d \lambda M_{3}(\lambda, \alpha) M_{3}^{*}\left(\lambda, \alpha^{\prime}\right)=\delta_{\alpha \alpha^{\prime}} \\
\int d \gamma M_{2}(\gamma, \beta) M_{2}^{*}\left(\gamma, \beta^{\prime}\right)+\int d \lambda M_{4}(\lambda, \beta) M_{4}^{*}\left(\lambda, \beta^{\prime}\right)=\delta_{\beta \beta^{\prime}} \\
\int d \gamma M_{1}(\gamma, \alpha) M_{2}^{*}(\gamma, \beta)+\int d \lambda M_{3}(\lambda, \alpha) M_{4}^{*}(\lambda, \beta)=0 .
\end{gathered}
$$

It is easily seen that the requirements (1.7) and (1.9) are meaningful if and only if the $M_{i}$ 's are all integral kernels of transformations of square integrable functions of the indices into other square integrable functions. This is also sufficient for the $B, B^{\dagger}, D, D^{\dagger}$ to be well defined operator valued distributions on all $\mathscr{H}$.

\section{Necessary Conditions for Existence of a New Vacuum}

We want to examine under what conditions a state $\left|\phi_{0}\right\rangle \in \mathscr{H}$ will exist such that

Writing

$$
B(\gamma)\left|\phi_{0}\right\rangle=0 \forall \gamma \quad \text { and } \quad D(\lambda)\left|\phi_{0}\right\rangle=0 \forall \lambda .
$$

$$
\left|\phi_{0}\right\rangle=\sum_{n=0}^{\infty} \sum_{m=0}^{\infty} \frac{1}{\sqrt{n ! m !}} \int \boldsymbol{d} \boldsymbol{\alpha}_{n} \boldsymbol{d} \boldsymbol{\beta}_{m} \psi^{n m}\left(\boldsymbol{\alpha}_{n} ; \boldsymbol{\beta}_{m}\right)\left|\boldsymbol{\alpha}_{n} ; \boldsymbol{\beta}_{m}\right\rangle
$$

and using $B(\gamma)$ as given in Eq. (1.1), we obtain that the following relations between the $\psi^{n m}$ 's must exist in order for $B(\gamma)\left|\phi_{0}\right\rangle$ to be null.

$$
\begin{aligned}
\int d \alpha_{j} M_{1}\left(\gamma, \alpha_{j}\right) \psi^{n m}\left(\boldsymbol{\alpha}_{n} ; \boldsymbol{\beta}_{m}\right)= & \frac{1}{\sqrt{m n}} \sum_{i=1}^{m}(-1)^{n+i+j} M_{2}\left(\gamma, \beta_{i}\right) \\
& \cdot \psi^{n-1 m-1}\left(\frac{\boldsymbol{\alpha}_{n}}{\hat{j}} ; \frac{\boldsymbol{\beta}_{m}}{\hat{i}}\right)
\end{aligned}
$$


for $n=1,2, \ldots, m=1,2, \ldots, \alpha_{j}$ being any one of the $\alpha_{n}$ and

$$
\int d \alpha_{j} M_{1}\left(\gamma, \alpha_{j}\right) \psi^{n 0}\left(\alpha_{n} ;-\right)=0 \text { for } n=1,2, \ldots
$$

Similarly, $D(\lambda)\left|\phi_{0}\right\rangle=0$ leads to

$$
\begin{aligned}
\int d \beta_{j} M_{4}^{*}\left(\lambda, \beta_{j}\right) \psi^{n m}\left(\boldsymbol{\alpha}_{n} ; \boldsymbol{\beta}_{m}\right)= & \frac{1}{\sqrt{m n}} \sum_{i=1}^{n}(-1)^{n+i+j+1} M_{3}^{*}\left(\lambda, \alpha_{i}\right) \\
& \cdot \psi^{n-1 m-1}\left(\frac{\boldsymbol{\alpha}_{n}}{\hat{i}} ; \frac{\boldsymbol{\beta}_{m}}{\hat{j}}\right)
\end{aligned}
$$

for $n=1,2, \ldots, m=1,2, \ldots, \beta_{j}$ being any one of the $\boldsymbol{\beta}_{m}$ and

$$
\int d \beta_{j} M_{4}^{*}\left(\lambda, \beta_{j}\right) \psi^{o m}\left(-; \boldsymbol{\beta}_{m}\right)=0 \text { for } m=1,2, \ldots
$$

We can find immediately a necessary condition for the Eqs. (2.3) and (2.5) to have square integrable solutions $\psi^{n m}$. [Square integrability of $\psi^{n m}$ for each $n$ and $m$ being required by relation (1.4).]

From the Eq. (1.9), we have

$$
\begin{aligned}
& \int d \gamma\left|\int d \alpha M_{1}(\gamma, \alpha) \psi(\alpha)\right|^{2} \leqq \int d \alpha|\psi(\alpha)|^{2} \\
& \int d \gamma\left|\int d \beta M_{2}(\gamma, \beta) \psi(\beta)\right|^{2} \leqq \int d \beta|\psi(\beta)|^{2} .
\end{aligned}
$$

Eq. (2.7) implies that $\psi^{n m}$ can be square integrable only if the right hand side of (2.3) is square integrable, i.e. we need

$$
\int d \gamma \frac{\boldsymbol{d} \boldsymbol{\alpha}_{n}}{\hat{j}} \boldsymbol{d} \boldsymbol{\beta}_{m}\left|\frac{1}{\sqrt{m n}} \sum_{i=1}^{m}(-1)^{n+i+j} M_{2}\left(\gamma, \beta_{i}\right) \cdot \psi^{n-1 m-1}\left(\frac{\boldsymbol{\alpha}_{n}}{\hat{j}} ; \frac{\boldsymbol{\beta}_{m}}{\hat{i}}\right)\right|^{2}<\infty .
$$

This is

$$
\begin{aligned}
\frac{1}{m n} \int d \gamma \frac{\boldsymbol{d} \boldsymbol{\alpha}_{n}}{\hat{j}} \boldsymbol{d} \boldsymbol{\beta}_{m} \sum_{i=1}^{m}\left|M_{2}\left(\gamma, \beta_{i}\right)\right|^{2}\left|\psi^{n-1 m-1}\left(\frac{\boldsymbol{\alpha}_{n}}{\hat{j}} ; \frac{\boldsymbol{\beta}_{m}}{\hat{i}}\right)\right|^{2} \\
+\frac{1}{m n} \sum_{\substack{i=1 \\
i \neq i^{\prime}}}^{m} \sum_{i^{\prime}=1}^{m}(-1)^{i+i^{\prime}} \int d \gamma \frac{\boldsymbol{d} \boldsymbol{\alpha}_{n}}{\hat{j}} \frac{\boldsymbol{d} \boldsymbol{\beta}_{m}}{\widehat{i t}^{\prime}} \\
\cdot\left(\int d \beta_{i} \psi^{n-1 m-1}\left(\frac{\boldsymbol{\alpha}_{n}}{\hat{j}} ; \frac{\boldsymbol{\beta}_{m}}{\hat{i}^{\prime}}\right)^{*} M_{2}\left(\gamma, \beta_{i}\right)\right) \\
\cdot\left(\int d \beta_{i^{\prime}} M_{2}^{*}\left(\gamma, \beta_{i^{\prime}}\right) \psi^{n-1 m-1}\left(\frac{\boldsymbol{\alpha}_{n}}{\hat{j}} ; \frac{\boldsymbol{\beta}_{m}}{\hat{i}}\right)\right)<\infty
\end{aligned}
$$

and supposing that $\psi^{n-1 m-1}$ was non-zero and square integrable; because of Eq. (2.8), the second integral above is finite so that in fact we need the first term to be finite, i.e.

$$
\int d \gamma d \beta\left|M_{2}(\gamma, \beta)\right|^{2}<\infty .
$$


Repeating the same argument with Eq. (2.5), we can see that we need also

$$
\int d \lambda d \alpha\left|M_{3}(\lambda, \alpha)\right|^{2}<\infty
$$

\section{Properties of the Operators $\boldsymbol{M}_{i}$}

Let us now suppose that the necessary conditions found in the preceding section are satisfied. We will examine some of the properties of the operators $M_{i}$.

i) The kernel $\left(M_{3}^{*} M_{3}\right)=\int d \lambda M_{3}^{*}\left(\lambda, \alpha^{\prime}\right) M_{3}(\lambda, \alpha)$ is a non-null hermitian $L^{2}$ kernel. It therefore has a spectral decomposition

$$
\left(M_{3}^{*} M_{3}\right)\left(\alpha^{\prime}, \alpha\right)=\sum_{n=1}^{\infty} \frac{1}{\Lambda_{n}^{3}} X_{n}^{3}\left(\alpha^{\prime}\right) X_{n}^{3}(\alpha)^{*}
$$

where $\left(\Lambda_{n}^{3}, X_{n}^{3}\right)$ is the characteristic system defined by

$$
\int d \alpha\left(M_{3}^{*} M_{3}\right)\left(\alpha^{\prime}, \alpha\right) X_{n}^{3}(\alpha)=\frac{1}{\Lambda_{n}^{3}} X_{n}^{3}\left(\alpha^{\prime}\right) .
$$

The Eq. (1.9a) implies $1 / \Lambda_{n}^{3}=1-\left\|M_{1} X_{n}^{3}\right\|^{2}$ and Eq. (3.2): $1 / \Lambda_{n}^{3}$ $=\left\|M_{3} X_{n}^{3}\right\|^{2}$ so that $0 \leqq 1 / \Lambda_{n}^{3} \leqq 1$. Since

$$
\int d \alpha d \alpha^{\prime}\left|\left(M_{3}^{*} M_{3}\right)\left(\alpha^{\prime}, \alpha\right)\right|^{2}=\sum_{n=1}^{\infty}\left(\frac{1}{\Lambda_{n}^{3}}\right)^{2}<\infty
$$

and since each characteristic value in this sequence is included a number of times equal to its rank, there is necessarily only a finite number of different $X_{n}^{33}$ s corresponding to a same given $\Lambda_{n}^{3}$. In particular, we shall call $N$ the number of eigenfunctions with eigenvalue 1 . We will choose the labels of the basis $\left\{X_{n}^{3}\right\}$ such that these correspond to $n=1,2, \ldots, N$. From $(1.9 \mathrm{a})$ it is easy to see that also

$$
\int d \alpha M_{1}(\gamma, \alpha) X_{n}^{3}(\alpha)=0 \forall \gamma \quad \text { for } n=1,2, \ldots, N .
$$

ii) Similarly

$$
\left(M_{3}^{*} M_{3}\right)\left(\lambda^{\prime}, \lambda\right)=\sum_{n=1}^{\infty} \frac{1}{\Lambda_{n}^{1}} X_{n}^{1}\left(\lambda^{\prime}\right) X_{n}^{1}(\lambda)^{*}
$$

and there is a finite number of $\Lambda_{n}^{1}$ which are equal to 1 . By $(1.7 \mathrm{~b}), X^{1}(\lambda)$ is such that

$$
\int d \lambda\left(M_{3}^{*} M_{3}\right)\left(\lambda^{\prime}, \lambda\right) X^{1}(\lambda)=X^{1}\left(\lambda^{\prime}\right)
$$

if and only if

$$
\int d \lambda M_{4}(\lambda, \beta) X^{1}(\lambda)=0 \forall \beta
$$


We shall now show that there are $N$ such functions. We do this by showing that such functions can be obtained from the $X_{n}^{3}, n=1,2, \ldots, N$ and vice versa.

For each one of the $X_{n}^{3}(\alpha), n=1,2, \ldots, N$, the function

$$
\left[\int d \alpha M_{3}(\lambda, \alpha) X_{n}^{3}(\alpha)\right]^{*}
$$

is easily seen to be non-zero. Such functions are orthogonal to each other; this follows from (3.2) where $\Lambda_{n}^{3}=1$ and the orthogonality of the $X_{n}^{3}$ 's. Each such function, because of $(1.9 \mathrm{c})$ is such that $\int d \lambda M_{4}(\lambda, \beta)\left[\int d \alpha M_{3}^{*}(\lambda, \alpha) X_{n}^{3}(\alpha)^{*}\right]=-\int d \gamma M_{2}(\gamma, \beta)\left[\int d \alpha M_{1}^{*}(\gamma, \alpha) X_{n}^{3}(\alpha)^{*}\right]$ and this is null by Eq. (3.3). For each $X_{n}^{3}(\alpha), n=1,2, \ldots, N$, there is then a

$$
X_{n}^{1}(\lambda)=\left[\int d \alpha M_{3}(\lambda, \alpha) X_{n}^{3}(\alpha)\right]^{*}
$$

such that this satisfies (3.6) or equivalently (3.5).

In turn the functions $X_{n}^{3}(\alpha), n=1,2, \ldots, N$ are related to $X_{n}^{1}$, $n=1,2, \ldots, N$ as:

$$
X_{n}^{3}(\alpha)=\left[\int d \lambda M_{3}(\lambda, \alpha) X_{n}^{1}(\lambda)\right]^{*} .
$$

iii) We shall need also the spectral decomposition of

which is

$$
\left(M_{2} M_{2}^{*}\right)\left(\beta^{\prime}, \beta\right)=\int d \gamma M_{2}\left(\gamma, \beta^{\prime}\right) M_{2}^{*}(\gamma, \beta)
$$

$$
\left(M_{2} M_{2}^{*}\right)\left(\beta^{\prime}, \beta\right)=\sum_{n=1}^{\infty} \frac{1}{\Lambda_{n}^{2}} X_{n}^{2}\left(\beta^{\prime}\right) X_{n}^{2}(\beta)^{*},
$$

with $0 \leqq 1 / \Lambda_{n}^{2} \leqq 1$ and we call $M(<\infty)$ the number of orthogonal $X_{n}^{2}$ which correspond to $\Lambda_{n}^{2}=1$. We have for these, from (1.9b):

$$
\int d \beta M_{4}^{*}(\lambda, \beta) X_{n}^{2}(\beta)=0 \vee \lambda \text { for } n=1,2, \ldots, N .
$$

We also have

$$
\left(M_{2} M_{2}^{*}\right)\left(\gamma^{\prime}, \gamma\right)=\sum_{n=1}^{\infty} \frac{1}{\Lambda_{n}^{4}} X_{n}^{4}\left(\gamma^{\prime}\right) X_{n}^{4}(\gamma)^{*}
$$

where the first $M X_{n}^{4}$ 's correspond to $\Lambda_{n}^{4}=1$ and they satisfy

$$
\int d \gamma M_{1}^{*}(\gamma, \alpha) X_{n}^{4}(\gamma)=0 \vee \alpha \text { for } n=1,2, \ldots, M .
$$

They are related to the $X_{n}^{2}$ s $n=1,2, \ldots, M$ according to:

and

$$
X_{n}^{4}(\gamma)=\left[\int d \beta M_{2}^{*}(\gamma, \beta) X_{n}^{2}(\beta)\right]^{*} \quad n=1,2, \ldots, M
$$

$$
X_{n}^{2}(\beta)=\left[\int d \gamma M_{2}^{*}(\gamma, \beta) X_{n}^{4}(\gamma)\right]^{*} \quad n=1,2, \ldots, M .
$$




\section{The Unitary Operator}

i) In order to see more clearly what happens in the transformation (1.1), we decompose it in a product of simpler transformations. We shall consider here the effect of the transformation on the following smeared creation and annihilation operators:

$$
\begin{array}{ll}
b_{n}=\int d \alpha X_{n}^{3}(\alpha)^{*} b(\alpha) & d_{n}^{\dagger}=\int d \beta X_{n}^{2}(\beta) d^{\dagger}(\beta) \\
B_{n}=\int d \gamma X_{n}^{4}(\gamma)^{*} B(\gamma) & D_{n}^{\dagger}=\int d \lambda X_{n}^{1}(\lambda) D^{\dagger}(\lambda) .
\end{array}
$$

We recall that the functions $X_{n}^{1}(\lambda), X_{n}^{2}(\beta), X_{n}^{3}(\alpha), X_{n}^{4}(\gamma)$ form bases in $L^{2}(\lambda), L^{2}(\beta), L^{2}(\alpha), L^{2}(\gamma)$ respectively. Clearly, a complete description of the transformation can be obtained by examining its effect on the operators $b_{n}, d_{n}, B_{n}, D_{n}$ for all values of the index $n$ labelling the elements of any arbitrary bases in $L^{2}(\lambda), L^{2}(\beta), L^{2}(\alpha), L^{2}(\gamma)$. In particular, it is obvious that $\left|\phi_{0}\right\rangle$ is such that

$$
B(\gamma)\left|\phi_{0}\right\rangle=0 \forall \gamma \quad \text { and } \quad D(\lambda)\left|\phi_{0}\right\rangle=0 \vee \lambda
$$

if and only if

$$
B_{n}\left|\phi_{0}\right\rangle=0 \vee n \quad \text { and } \quad D_{n}\left|\phi_{0}\right\rangle=0 \vee n
$$

when $n$ labels bases in $L^{2}(\gamma)$ and $L^{2}(\lambda)$.

The inverse relations to (4.1) are

$$
\begin{array}{ll}
b(\alpha)=\sum_{n=1}^{\infty} X_{n}^{3}(\alpha) b_{n} & d^{\dagger}(\beta)=\sum_{n=1}^{\infty} X_{n}^{2}(\beta)^{*} d_{n}^{\dagger} \\
B(\gamma)=\sum_{n=1}^{\infty} X_{n}^{4}(\gamma) B_{n} & D^{\dagger}(\lambda)=\sum_{n=1}^{\infty} X_{n}^{1}(\lambda)^{*} D_{n}^{\dagger} .
\end{array}
$$

Because of the properties of the functions $X_{n}^{1}$ which we have described in Section 3), one can see that the Bogoliubov transformation (1.1) is in fact:

$$
\begin{array}{r}
B_{n}=d_{n}^{\dagger} \text { for } n=1,2, \ldots, M \quad D_{n}^{\dagger}=b_{n} \text { for } n=1,2, \ldots, N \\
B_{M+n^{\prime}}=\sum_{n=1}^{\infty}\left\{\left(X_{M+n^{\prime}}^{4}, M_{1} X_{N+n}^{3}\right) b_{N+n}+\left(X_{M+n^{\prime}}^{4}, M_{2} X_{M+n}^{2}\right) d_{M+n}^{\dagger}\right\} \\
D_{N+n^{\prime}}^{\dagger}=\sum_{n=1}^{\infty}\left\{\left(X_{N+n^{\prime}}^{1}, M_{3} X_{N+n}^{3}\right) b_{N+n}+\left(X_{N+n^{\prime}}^{1}, M_{4} X_{M+n}^{2}\right) d_{M+n}^{\dagger}\right\} \\
\text { for } n^{\prime}=1,2, \ldots, \infty .
\end{array}
$$

The Eq. (4.4) itself is a Bogoliubov transformation between the two sets of operators $\tilde{b}_{n}=b_{N+n}, \tilde{d}_{n}=d_{M+n} n=1,2, \ldots, \infty$, and $\tilde{B}_{n}=B_{M+n}$, 
$\tilde{D}_{n}=D_{N+n} n=1,2, \ldots, \infty$. The kernels $\tilde{M}_{1}\left(n^{\prime}, n\right) \equiv\left(X_{M+n^{\prime}}^{4}, M_{1} X_{N+n}^{3}\right)$ and $\tilde{M}_{4}\left(n^{\prime}, n\right)^{*} \equiv\left(X_{M+n}^{2}, M_{4}^{*} X_{N+n^{\prime}}^{1}\right)$ do not have any non-zero homogeneous solutions associated with them as is easily deduced from the properties of the $X_{n}^{i \text { s. }}$. (4.4) is therefore a "weak" Bogoliubov transformation.

ii) Let us now look more particularly at "weak" Bogoliubov transformations. We recall that the unitary operators implementing such transformations are known (for the form used below, see Section 24 of the Part V of Friedrichs' book [1]). They can be written, for example, as

$$
T=\eta e^{-d F_{4} b} e^{-d^{\dagger} F_{3} d} e^{-b^{\dagger} F_{2} b} e^{-b^{\dagger} F_{1} d^{\dagger}}
$$

where expressions like $e^{-b^{\dagger} F_{1} d^{\dagger}}$ stand for

$$
\exp \left\{-\sum_{n^{\prime}=1}^{\infty} \sum_{n=1}^{\infty} b_{n^{\prime}}^{\dagger} F_{1}\left(n^{\prime}, n\right) d_{n}^{\dagger}\right\}
$$

and $\eta=\exp 1 / 2\left(\right.$ Trace $F_{2}+$ Trace $\left.F_{3}\right)$ has finite norm.

The $F_{i}$ 's are given by

$$
\begin{aligned}
& \left(e^{-F_{2}}\right)(n, m)=M_{1}(n, m) \\
& \left(e^{-F_{3}}\right)(n, m)=\left[M_{4}(n, m)\right]^{*} ;
\end{aligned}
$$

$F_{1}(n, m)$ is the solution of

and $F_{4}(n, m)$ of

$$
\sum_{n=1}^{\infty} M_{1}\left(n^{\prime}, n\right) F_{1}(n, m)=-M_{2}\left(n^{\prime}, n\right)
$$

$$
\sum_{n^{\prime}=1}^{\infty} M_{1}\left(n^{\prime}, n\right) F_{4}\left(m, n^{\prime}\right)=-M_{3}(m, n) .
$$

iii) In the case of the general transformation (4.3), (4.4), we can then define a unitary operator $T_{0}$ such that

and

$$
\begin{aligned}
& T_{0}^{\dagger} b_{N+n} T_{0}=B_{M+n} \\
& T_{0}^{\dagger} d_{M+n} T_{0}=D_{N+n}
\end{aligned} \quad \forall n=1,2, \ldots, \infty
$$

$$
\begin{array}{ll}
T_{0}^{\dagger} b_{i} T_{0}=b_{i} & \text { for } \quad i=1,2, \ldots, N, \\
T_{0}^{\dagger} d_{i} T_{0}=d_{i} & \text { for } \quad i=1,2, \ldots, M .
\end{array}
$$

That is: $T_{0}$ implements the "weak" Bogoliubov transformation (4.4) so that it exists as we saw in the previous section. It does not contain the operator variables not entering in (4.4) because of the requirement (4.9). It can be written in the form (4.5) for example, where the operators $(b, d)$ are $\tilde{b}_{n}, \tilde{d}_{n} ; n=1,2, \ldots$ and the $M_{i}$ 's are the $\tilde{M}_{i}$ 's. 
It is easy to check that the unitary operator

$$
\begin{aligned}
U= & \left\{\exp -i(M+N) \pi \sum_{n=1}^{\infty}\left[b_{n}^{\dagger} b_{n}+d_{n}^{\dagger} d_{n}\right]\right\}\left(d_{M}^{\dagger}-d_{M}\right) \ldots \\
& \ldots\left(d_{2}^{\dagger}-d_{2}\right)\left(d_{1}^{\dagger}-d_{1}\right)\left(b_{N}^{\dagger}-b_{N}\right)\left(b_{2}^{\dagger}-b_{2}\right)\left(b_{1}^{\dagger}-b_{1}\right)
\end{aligned}
$$

commutes with $b_{N+n}$ and $d_{M+n}$ for $n=1,2, \ldots, \infty$ and

$$
U^{\dagger} b_{i} U=D_{i} \text { for } i=1,2, \ldots, N, \quad U^{\dagger} d_{i} U=B_{i} \text { for } i=1,2, \ldots, M .
$$

The unitary operator

$$
T=T_{0} U=U T_{0}
$$

will then transform the creation and annihilation operators as

$$
\begin{array}{llll}
T^{\dagger} b_{n} T=D_{n} & n=1,2, \ldots, N & T^{\dagger} b_{n} T=B_{n} & n=N+1, \ldots, \infty \\
T^{\dagger} d_{n} T=B_{n} & n=1,2, \ldots, M & T^{\dagger} d_{n} T=D_{n} & n=M+1, \ldots, \infty .
\end{array}
$$

The vacuum $\left|\phi_{0}\right\rangle$ (i.e. the new zero particle state) is then simply

$$
\begin{aligned}
\left|\phi_{0}\right\rangle & =T^{\dagger}|0\rangle=b_{1}^{\dagger} b_{2}^{\dagger} \ldots b_{N}^{\dagger} d_{1}^{\dagger} \ldots d_{M}^{\dagger} T_{0}^{\dagger}|0\rangle \\
& =b_{1}^{\dagger} b_{2}^{\dagger} \ldots b_{N}^{\dagger} d_{1}^{\dagger} \ldots d_{M}^{\dagger} \frac{1}{\eta_{0}} \exp \sum_{n^{\prime}=N+1}^{\infty} \sum_{n=M+1}^{\infty} b_{n^{\prime}}^{\dagger} F_{1}^{0}\left(n^{\prime}, n\right) d_{n}^{\dagger}|0\rangle
\end{aligned}
$$

where $F_{1}^{0}(n, m)$ is the unique solution of

$$
\begin{gathered}
\sum_{n=N+1}^{\infty}\left(X_{n^{\prime}}^{4}, M_{1} X_{n}^{3}\right) F_{1}^{0}(n, m)=-\left(X_{n^{\prime}}^{4}, M_{2} X_{m}^{2}\right) \\
\forall n^{\prime}=M+1, M+2, \ldots, \infty \text { and } m=M+1, M+2, \ldots, \infty
\end{gathered}
$$

and $\eta_{0}$ is the finite normalization constant corresponding to $\eta$ in Eq. (4.5).

\section{Conclusion}

We examine what it would mean if such "strong" Bogoliubov transformations happened in the description of dynamical systems.

Let us consider a quantum mechanical system where operators $b, b^{\dagger}, d, d^{\dagger}$ are associated with particles at some time $t_{1}$ (which could be finite or $-\infty)$ and $B, B^{\dagger}, D, D^{\dagger}$ are associated with the same particles at a later time $t_{2}$ (finite or $+\infty$ ); this, according to an equation of motion like (1.1) in the Heisenberg representation. We suppose that the Bogoliubov transformation is a "strong" transformation and is unitarily implementable.

i) The vacuum at time $t_{2}$ is orthogonal to the vacuum at time $t_{1}$. 
This obviously would "complicate" a calculation of $|0\rangle_{t_{2}}\left(\equiv\left|\phi_{0}\right\rangle\right)$ through a perturbation series (in powers of the coupling constant for example) which would start from $|0\rangle_{t_{1}}(\equiv|0\rangle)$. The same remark evidently holds about a calculation of the unitary operator implementing the Bogoliubov transformation (which is a time evolution operator $U\left(t, t^{\prime}\right)$ or the $S$-matrix if $t_{1}$ is $-\infty$ and $t_{2}$ is $+\infty$ ) through a series starting with $I$.

ii) There are states of the system in which particles and antiparticles will be created with probability 1 .

If the system is in the state $|0\rangle_{t}$, there are no particles present initially. To see what this state represents at time $t_{2}$, we observe that the state with zero particles at this time is $|0\rangle_{t_{2}}$. The state $T_{0}|0\rangle_{t_{2}}$ is one in which there is a non-zero probability of finding pairs of particles and antiparticles created by $B_{M+n}^{\dagger}$ and $D_{M+n}^{\dagger} n=1,2, \ldots, \infty$. The state $|0\rangle_{t_{1}}$, which is

$$
|0\rangle_{t_{1}}=T|0\rangle_{t_{2}}=B_{M}^{\dagger} \ldots B_{2}^{\dagger} B_{1}^{\dagger} D_{N}^{\dagger} \ldots D_{1}^{\dagger} T_{0}|0\rangle_{t_{2}},
$$

is then a state in which $M$ particles and $N$ antiparticles have been created and in which there is a non-zero probability of finding also pairs of particles and antiparticles.

If, on the other hand, the system is in the state $|0\rangle_{t_{2}}$;

$$
|0\rangle_{t_{2}}=b_{1}^{\dagger} b_{2}^{\dagger} \ldots b_{N}^{\dagger} d_{1}^{\dagger} \ldots d_{M}^{\dagger} T_{0}^{\dagger}|0\rangle_{t_{1}},
$$

initially there are $N$ particles and $M$ antiparticles plus possibly some particle-antiparticle pairs. After the interaction, there are no particles present.

iii) If the particles and antiparticles are assigned a different charge, the total charge is not conserved unless $N=M$.

The charge operator at the different times is:

and

$$
Q\left(t_{1}\right)=e \sum_{n=1}^{\infty}\left\{b_{n}^{\dagger} b_{n}-d_{n}^{\dagger} d_{n}\right\}
$$

$$
Q\left(t_{2}\right)=e \sum_{n=1}^{\infty}\left\{B_{n}^{\dagger} B_{n}-D_{n}^{\dagger} D_{n}\right\} .
$$

By examining the action of these operators on any one of the two Fock bases (obtained by the action of $b^{\dagger}, d^{\dagger}$ on $|0\rangle_{t_{1}}$ or of $B^{\dagger}, D^{\dagger}$ on $|0\rangle_{t_{2}}$ ), one can see that they are related as

$$
Q\left(t_{2}\right)=Q\left(t_{1}\right)+e(M-N) .
$$

It is easy to see that the results obtained apply also to the case of one operator variable transformations like

$$
B(\gamma)=\int d \alpha M_{1}(\gamma, \alpha) b(\alpha)+\int d \alpha M_{2}(\gamma, \alpha) b^{\dagger}(\alpha) .
$$


In terms of smeared operators defined similarly as in the case discussed here, this transformation reduces to

$$
B_{n}=b_{n}^{\dagger} \quad n=1,2, \ldots, M
$$

and an ordinary Bogoliubov transformation between the other $B_{M+n}$ 's and $b_{M+n}$ 's. The unitary operator relating then has the form $T=T_{0} U$ where $T_{0}$ is the usual one for the "weak" transformation and

$$
U=\left\{\exp -i M \pi \sum_{n=1}^{\infty} b_{n}^{\dagger} b_{n}\right\}\left(b_{M}^{\dagger}-b_{M}\right) \ldots\left(b_{2}^{\dagger}-b_{2}\right)\left(b_{1}^{\dagger}-b_{1}\right)
$$

It is to be remarked that for a Bogoliubov transformation on a system of bosons or for a "weak" transformation with fermions, $N$ and $M$ above are always null. What we have described in i), ii), and iii) would then never happen in these cases.

We finally note that Akhiezer and Berestetsky have indicated (in the discussion at the end of Section $\S 17$ of their book [8]) that external electromagnetic field theory could predict creation (or annihilation) of single electrons or positrons. This would happen when one does not restrict appropriately the strength of the potential which he switches on. For strong potentials, what gives rise to this is that some positive (negative) energy wave functions are transformed completely by the $c$-number evolution operator into negative (positive) energy wave functions. This can be prevented by demanding a condition like $\left|\left(\psi, e \gamma^{0} \gamma A \psi\right)\right|$ $<\left(\psi,\left|\gamma^{0}(i \gamma \cdot \partial+m)\right| \psi\right) \forall \psi$ for example. (This is the condition which leads to $Z \leqq 87$ for the Coulomb potential $Z e / r$; this is discussed in the Chapters V and VI of Kato's book [9].)

It would be interesting to examine other existing external field theories to see how a similar "weakness" condition comes in.

Acknowledgements. I am grateful to Professor A. Z. Capri for very helpful discussions and encouragements in solving this problem. I want to thank Professor A. S. Wightman whose judicious comments helped me improve the final state of this note. I am also grateful for the hospitality of Princeton University.

\section{References}

1. Friedrichs, K.O.: Mathematical aspects of the quantum theory of fields. New York: Interscience Publishers, Inc. 1953

2. Capri, A.Z.: J. Math. Phys. 10, 575 (1969)

3. Schroer, B., Seiler, R., Swieca,J.A.: Phys. Rev. 2D, 2927 (1970)

4. Seiler, R.: Commun. math. Phys. 25, 127 (1972) 
5. Shale,D.: Transact. Amer. Math. Soc. 103, 149 (1962)

6. Bongaarts, P.J. M.: Ann. Phys. 56, 108 (1970)

7. Shale,D., Stinespring, W.F.: J. Math. and Mech. 14, 315 (1965)

8. Akhiezer,A.I., Berestetsky,V.B.: Quantum electrodynamics. Translation for the Atomic Energy Comm. U.S.A., Dept. of Commerce, Washington 25, D.C. (1953)

9. Kato,T.: Perturbation theory for linear operators. Berlin-Heidelberg-New York: Springer-Verlag 1966

Communicated by A. S. Wightman

Gilles Labonté

Theoretical Physics Institute

University of Alberta

Edmonton, Canada

Present address:

Joseph Henry Laboratories of Physics

Princeton University

Princeton, N.J. 08540, USA 